\title{
CRISPR/Cas9 Gene Editing Therapies for Cystic Fibrosis
}

\section{Carina Graham \& Stephen Hart}

To cite this article: Carina Graham \& Stephen Hart (2021): CRISPR/Cas9 Gene Editing Therapies for Cystic Fibrosis, Expert Opinion on Biological Therapy, DOI: 10.1080/14712598.2021.1869208

To link to this article: https://doi.org/10.1080/14712598.2021.1869208

Accepted author version posted online: 08 Jan 2021.

Submit your article to this journal $\square$

Q View related articles 두

View Crossmark data \lceil 
Publisher: Taylor \& Francis \& Informa UK Limited, trading as Taylor \& Francis Group

Journal: Expert Opinion on Biological Therapy

DOI: $10.1080 / 14712598.2021 .1869208$

\title{
CRISPR/Cas9 Gene Editing Therapies for Cystic Fibrosis
}

Carina Graham, Stephen Hart

University College London Institute of Child Health

\section{Article Highlights}

- Cystic fibrosis (CF) is a common life-limited genetic disorder that has historically resisted gene-based treatments.

- Recent advances in pharmacological treatment of CF are promising for patients harbouring specific genotypes, but many patients' genotypes remain "undruggable."

- The advent of CRISPR-Cas9 technology in the mid-2010s vastly broadened the possibilities for targeted gene editing in mammalian cells.

- CRISPR-Cas9 has been used in recent years to correct several mutations causing CF, yielding significant rescue of electrophysiological defects in modelled human epithelia and organoids.

- Technologies derived from CRISPR-Cas9, including base editing, prime editing, and homology-independent targeted integration (HITI) can also potentially be adapted to create novel CF treatments.

\begin{abstract}
Introduction: Cystic fibrosis (CF) is a life-limiting genetic disorder affecting approximately 70,000 people worldwide. Current burden of treatment is high. While the latest pharmaceutical innovation has benefitted many, patients with certain genotypes remain excluded. Gene editing has the potential to correct the underlying cause of disease for all patients, representing a permanent cure.
\end{abstract}

Areas covered: Various DNA editing-based strategies for treatment are currently being developed. Different strategies are called for based upon location of mutations (intronic vs. exonic), delivery mechanism of editing machinery, and cell type being targeted. Furthermore, 
the unique physiology of the CF lung presents a variety of barriers to delivery of CRISPRCas9 machinery.

Expert opinion: The most significant obstacle to the use of CRISPR-Cas9 in vivo is the fact that the most clinically relevant and accessible CF tissue, the airway epithelium, is made up of non-dividing cells where precise editing via homology-directed repair (HDR) does not occur; rather, potentially deleterious imprecise editing via non-homologous end joining (NHEJ) dominates. Future research should focus on the development of either more precise NHEJ-based approaches, access to airway basal cells, editing approaches that do not involve introducing genomic double strand breaks, and strategies with ex vivo edited cells.

Keywords: cystic fibrosis, CRISPR-Cas9, CRISPR, gene editing, lung, airway, genetic disease, DNA, prime editing, base editing

\section{Introduction}

\subsection{Cystic Fibrosis}

Cystic fibrosis (CF) is an autosomal recessive disorder estimated to affect 70,000 people worldwide [1, 2]. In 2018, the median life expectancy for UK-born homozygotes of the most common CF mutation was 43.5 years of age [3]. However, in less developed countries, life expectancy drops to under 15 years [4]. CF is caused by defects in the protein CFTR (cystic fibrosis transmembrane conductance regulator), encoded by the CFTR gene. CFTR forms a chloride and bicarbonate ion channel on the apical surface of airway epithelial cells, allowing chloride ions into the airway surface liquid (ASL), which comprises the mucus layer above the periciliary liquid layer (PCL) $[2,5,6]$. Ion flow creates osmotic pressure, which draws water into the ASL to maintain the thin, watery consistency of the PCL. When the CFTR protein is non-functional, chloride ions are retained inside epithelial cells. Simultaneously, loss of CFTR-mediated inhibition of the epithelial sodium channel, $\mathrm{ENaC}$, leads to sodium hyperabsorption; this phenomenon is associated with transepithelial and paracellular uptake of water from the ASL, leading to ASL dehydration $[2,5,6]$.

Complications arising from lung disease are the major cause of mortality in CF [7]. Disease arises due to multiple causes. Firstly, thick, sticky mucus clogs airways and impedes gas exchange [1, 2, 7-9]. Secondly, airway cilia motility is impeded by mucus, and cilia are unable to sweep pathogens and debris out of the lungs $[5,10]$. Thirdly, impaired ion transport 
has been implicated in a decrease in ASL pH, which inhibits antimicrobial peptide defences in the airways [11-17].

Inhibition of pathogen clearance via mucociliary clearance, and loss of antimicrobial activity in the ASL, causes chronic persistent bacterial infection [8] leading to recruitment of neutrophils into the lung [18-20]. Accumulation of DNA released from dead neutrophils causes further thickening of mucus [9] while prolonged release of neutrophil elastase damages the collagen matrices of the lungs [18-20]. Tissue damage then triggers further responses from the immune system, leading to a feedback effect of chronic inflammation [20], which in turn further restricts airflow. In order to combat chronic bacterial infection, CF patients are often given prolonged treatments with antibiotics such as aminoglycosides [21] which, on extended use, can cause nephrotoxic, vestibulotoxic, and ototoxic side-effects [21].

There are over 2,000 known genetic variants of CFTR, and over 300 of these are known to cause CF $[22,23]$. Researchers have categorized mutations into six classes. Class I mutations result in complete prohibition of protein production. Class II mutations result in inappropriate trafficking of CFTR to the apical surface of epithelial cells. Class III mutations result in impairment of ion channel gating while Class IV mutations result in decreased flow of ions through the CFTR channel. Class V mutations result in diminished quantity of protein produced and Class VI mutations result in decreased retention of protein at the cell surface [1, 2, 24]. The most common CF-causing mutation, F508del, is commonly classified as a Class II mutation, as its resulting malformed protein is typically trafficked for endoplasmic reticulumassociated degradation; however, F508del protein that escapes degradation also exhibits characteristics of Class II and VI mutations (gating defects and rapid turnover, respectively) $[1,2,24,25]$. F508del consists of a three base-pair deletion resulting in loss of a phenylalanine residue. An estimated $90 \%$ of patients carry at least one copy of this allele [26].

In the last ten years, there has been significant progress in healthcare for CF patients. It is now estimated that the majority of living CF patients are adults, and anticipated that life expectancy will continue to increase [3]. The recently developed triple-combination drug ivacaftor-tezacaftor-elexacaftor promises to improve quality of life and life expectancy for any CF patient who carries at least one copy of the F508del allele [26-28]. The triple combination drug works by combining three small molecule approaches. Firstly, two "corrector" molecules (Elexacaftor ${ }^{\mathrm{tm}}$ and Tezacaftor ${ }^{\mathrm{t}}$ ) aid in proper trafficking and localization of CFTR protein in the apical membrane of the epithelial cells for conformationaltering mutations. Secondly, a "potentiator" (Ivacaftor ${ }^{\mathrm{tm}}$ ) increases activity of CFTR at the 
cell surface $[27,28]$. In clinical trials, patients treated with triple-combination drug showed significant improvement in forced expiratory volume in one second (FEV1), decreases in pulmonary exacerbations, and reductions in sweat chloride concentration (all common diagnostic measurements of cystic fibrosis disease progression and severity) when compared with a placebo [27].

However, there are many mutations that remain untreatable by pharmaceutical approaches. For example, $10 \%$ of CF patients carry mutations resulting in truncated or misspliced protein products whose folding and trafficking cannot be rescued by the drug [29]. For patients with these alleles, the burden of treatment is extremely high. As of 2019, the mean reported time spent engaged in some form of treatment activity was two hours a day [30]. Treatments focused on clearing the airway of mucus often involve time-consuming and physically uncomfortable activities which can have a serious negative impact on patients' quality of life. Additionally, $76 \%$ of patients with cystic fibrosis and their carers reported obstruction in obtaining necessary medications, so even those eligible for treatment with triple-combination drug may face difficulty in acquiring it [30]. In the case of patients who are able to obtain and take the triple-combination drug, a clinical trial is currently underway to determine whether or not it is safe to cease other concurrent treatments; until that has been determined, burden of care will remain high even for patients with access to the drug [31, 32].

Gene therapy approaches to CF offer an alternative approach for patients with undruggable mutations but have so far yielded mixed success in clinical trials with both viral and non-viral approaches. Multiple adenoviral vector approaches were explored in CF gene therapy trials between 1989 and 2001 [33]. However, the non-integrating nature of adenoviruses yields transient expression, requiring repeated administration, which is limited by a strong antiviral immune response [34]. Adeno-associated viruses (AAV) are generally regarded as safer than adenoviruses and can give longer term expression, even without integration, but their limited packaging capacity $(\sim 4.7 \mathrm{~kb})$ precludes the use of promoters or enhancers alongside lengthy CFTR cDNA ( $4.5 \mathrm{~kb})$; a 1998 clinical trial using AAV vectors failed to achieve its primary endpoints [33]. However, in recent years the evaluation of new serotypes and engineered capsids with lung tropism has led to a revived interest in AAVmediated CF gene therapy; however, anti-viral immune response remains problematic [35]. Alternatively, lentiviral vectors' ability to integrate into the host genome may reduce the need for frequent repeated dosage to the lung, while alternative pseudotypes can enhance cellular tropism to the lung [35]. A 2017 preclinical study involving lentiviral vectors for CF 
treatment showed high efficacy and low toxicity with a simian immunodeficiency virus which was pseudotyped with Sendai virus envelope fusion and hemagglutinin proteins to mediate membrane fusion [36].

Non-viral approaches to CF gene therapy, including lipid nanoparticles, have been extensively evaluated; the most recent study was a randomised, double-blind, placebocontrolled, phase $2 \mathrm{~b}$ clinical trial in which a CFTR cDNA plasmid encapsulated in cationic nanoparticles was delivered by nebulization at monthly intervals for one year [37]. Treatment led to stabilization, but not improvement, of the FEV1 in treated patients relative to those receiving a placebo.

Transient and low levels of CFTR transgene expression underpin the problems encountered in gene therapy trials to date, and so alternative approaches are required. Recently CRISPR gene editing technologies have emerged with the potential for precise and permanent correction of cells [38], opening up opportunities for editing of airway epithelial cells for CF.

\subsection{Gene Editing with CRISPR-Cas9}

CRISPR (Clustered Regularly Interspaced Short Palindromic Repeats) is a powerful gene editing tool, adapted from a bacterial mechanism for adaptive immunity [39-43]. When foreign genetic material is detected by bacterial species such as Streptococcus pyogenes, a portion of the invading sequence is copied into a part of the host genome known as a CRISPR array. When transcribed, this array generates multiple RNAs that have homology to invading bacteriophages' genetic material [40, 42, 43]. Each of these CRISPR RNAs (crRNAs) then associates with a nuclease protein, such as Cas9; this association is aided by a scaffolding trans-activating small RNA (tracrRNA). When a CRISPR-Cas9 ribonucleoprotein (RNP) complex discovers a piece of DNA homologous to the crRNA, the Cas9 binds to and creates a double-strand break in the invading DNA, just upstream of a protospacer-adjacent motif (PAM) site [39-43].

This naturally occurring system of bacterial adaptive immunity has since been adapted into a technology allowing efficient and precise DNA editing in both mammalian and non-mammalian cells [38]. By synthesizing custom crRNAs with homology to target loci, researchers can direct Cas9 to form a double-stranded break (DSB) in any desired sequence of DNA [39, 44, 45]. 
Because DSB formation threatens genome stability and cell health, swift and efficient repair is vital [46-49]. Cells typically use one of two methods to repair DSBs. One is the nonhomologous end-joining (NHEJ) pathway $[46-48,50]$ in which the Ku protein heterodimer binds exposed ends of the DNA and recruits further molecular machinery; these molecules stabilize the DNA while DNA ligase IV rejoins the cut ends [46, 50]. Often during NHEJ, random deletion or insertion of nucleotides ("indels") occurs at the cut ends during repair [48, 50]. Another commonly occurring DNA repair pathway, homology-directed repair (HDR), is much more precise. HDR uses the homologous chromosome, or experimenter-provided DNA, as a template to rebuild the cut DNA $[41,51,52]$. Reconstruction can occur unidirectionally in the upstream or downstream direction, or bidirectionally [53]. This allows researchers to incorporate precise changes at the single nucleotide level or incorporate large insertions such as marker genes.

NHEJ is useful for generating knockouts of genes, as it often results in frameshifts arising from random deletions and insertions, or in large unwanted deletions and rearrangements [54]. Additionally, by using two guide RNAs distant from one another, it can be used to excise large portions of a gene [45]. For small, exact edits to the genome (such as correction of disease-causing single nucleotide polymorphisms (SNPs)), HDR is preferred for its precision $[52,55]$ although this pathway is only active during the G2 and S phases of the cell cycle [52, 56], rendering it inactive in non-dividing cells. Even in actively dividing cells, the NHEJ pathway remains more active than HDR, leading to simultaneous indel formation in some alleles, while the intended HDR event may occur in others [52].

\section{Delivery of CRISPR-Cas9 Machinery}

\subsection{Plasmid Delivery Approaches}

The first experiments with CRISPR-Cas9 in mammalian cells utilized codonoptimized DNA expression constructs in plasmids to express Cas9 and gRNAs [38, 44]. Such approaches have been used in studies of gene editing for CF. In one such study, cells from a CF F508del-homozygous patient were expanded and reprogrammed into induced pluripotential stem cells (iPSCs) [57]. iPSCs were nucleofected with codon-optimized Cas9 encoded in a plasmid under the Eukaryotic Translation Elongation Factor 1 Alpha 1 (EEF1A1) promoter, while gRNAs targeting the F508del mutation region were encoded in a plasmid under the U6 promoter [57]. Donor vectors included template for correction of the F508del mutation and puromycin selection marker [57]. After puromycin selection, surviving 
cells were screened via PCR for proper location and orientation of donor integration. 6 of the 36 screened puromycin-resistant clones were correctly edited in at least one allele [57].

In another study, intestinal organoids derived from two CF patients, each homozygous for the F508del mutation [58], were transfected with plasmids encoding both Cas9 and gRNAs targeting the F508 locus, as well as a puromycin-resistance gene to enable selection of corrected cells [58]. The study showed that $18 \%$ of puromycin-resistant transfected organoids were successfully edited, although transfection efficiency was only $1.5 \%$.

Plasmid DNA is a reasonably effective method of delivering Cas9 and gRNAs; however, it comes with several disadvantages. Firstly, there is the delay in expression due to the time it takes for protein expression of Cas9 and RNA expression of gRNA cassettes. Secondly, plasmids yield longer-term expression of Cas9, which can increase the risk of offtarget DSBs, creating unwanted indels and risk of apoptosis [59-61]. Exacerbating this risk is the potential for plasmid DNA to integrate into the host genome [60]. Thirdly, in both studies discussed above, plasmids included cassettes selecting for antibiotic resistance to aid in selection of edited cells; use of antibiotic resistance selection markers is commonly discouraged in clinical settings and in vivo research [62].

\subsection{Cas9 mRNA and RNP Approach to CRISPR/Cas9 Delivery}

In order to avoid the drawbacks of plasmid delivery, Cas9 may be delivered as in vitro transcribed messenger RNA (mRNA) mixed with synthetic gRNA, or as purified Cas9 protein pre-loaded with gRNA in a ribonucleoprotein complex (RNP) [60,63]. In addition to the transient activity of purified mRNA and RNPS, these approaches enable the use of synthetic gRNAs which may be chemically modified to greatly enhance targeting efficiency and reduce cytotoxicity [64-67].

Messenger RNA and RNPs may be transfected into cells via electroporation, encapsulation in cell-permeating nanoparticles, or microinjection [68]. RNP transfection efficiency outperforms plasmid-encoded Cas9/gRNAs transfection in multiple cell types, including human iPSCs $[60,69]$. Transfection of RNPs via electroporation has been shown to efficiently cut the CFTR gene at the F508, G542, and G551 loci in wild-type iPSCs, with indel formations of $30 \%, 51 \%$, and $48 \%$ respectively [69]. In human upper and lower airway stem cells, electroporation of optimized RNP yielded over $80 \%$ cutting (resulting in $38 \%$ indel formation and 43\% homologous recombination) at the F508del mutant locus [70].

In vivo delivery of Cas9 mRNA and gRNAs can be accomplished using cellpermeating lipid nanoparticles with high tropism to lung cells and tissues both in vitro and in 
vivo. For example, selective organ targeting (SORT) nanoparticles incorporating the cationic lipids dimethyldioctadecylammonium (DDAB) and 1,2-dimyristoyl-sn-glycero-3ethylphosphocholine (EPC) achieved 40\% transfection of Cre-mRNA in Floxed TdTomato transgenic mouse lung epithelial cells [71]. Zwitterion amino lipid (ZAL) nanoparticles have been used to deliver Cas9 mRNA and guide RNAs to lung cancer A549 cells in vitro (as measured by Cas 9 mRNA content relative to actin mRNA content over 45 hours) and Rosa26 TdTomato transgenic mouse lungs in vivo (measured by fluorescence after Cas9-mediated removal of a STOP codon upstream of TdTomato in the cassette) [72]. Using the same TdTomato stop-removal reporter system, increased lung-specific fluorescence was achieved using CRISPR RNP encapsulated in a lipid nanoparticle formation consisting of the ionizable cationic lipid 5A2-SC8 formulated with 60\% 1,2-dioleoyl-3-trimethylammonium-propane (DOTAP) [73]. Additionally, nanoparticles can be conjugated with peptides that facilitate cell penetration and/or targeting; for example, a peptide identified by biopanning of a phage peptide library was recently used to successfully target nanocomplexes to lung epithelial cells [74].

\section{Delivery of Repair Template}

\subsection{DNA Oligonucleotides as Donor DNA}

In dividing cells, homology-directed repair (HDR) of Cas9-induced DNA damage can be achieved using a single-stranded oligodeoxynucleotide (ssODN). In this approach, DNA containing the desired edit is flanked by "arms" of sequence homologous to the genome flanking the cut site. These homology arms direct incorporation of the modified sequence into the repair of the DSB. In a recent study, pairing Cas9 RNP with ssODN repair enabled researchers to both create and repair common CF mutations in human iPSCs [69]. In order to induce CFTR mutations in wild-type (WT) cells, Cas9 RNP incorporating gRNAs targeting F508, G542, or G551 were electroporated into WT iPSCs. Simultaneously to this RNP construct, ssODN repair templates were electroporated. To induce the F508del mutation in WT iPSCs, sense or antisense ssODN templates were compared, both of which carried nearsymmetrical homology arms of 50 and 52 bases. The sense template yielded an editing efficiency of $5 \%$, while the antisense template yielded editing efficiency of $<1 \%$ [69]. To induce the G551D mutation in WT iPSCs, ssODN sense templates with asymmetrical homology arms of 118 and 82 bases had an editing efficiency of 19\% [69] while, to induce the G542X mutation in WT iPSCs, ssODN sense template with symmetrical homology arms of $80 \mathrm{bp}$ had an editing efficiency of $27 \%$ [69]. To repair the F508del mutation in patient- 
derived F508del homozygous iPSCs, sense or antisense ssODNs carried near-symmetrical homology arms of 50 and 52 bases; both sense and antisense repairs yielded editing efficiencies of $12 \%$ [69].

Overall, in this study, repair efficiency was inconsistent, and did not strongly correlate with symmetry, length, or sense vs. antisense homology. However, controlled direct comparison of such design factors were not explored for the same alleles. Notably, studies in optimization of ssODN design have suggested that both length and degree of symmetry in ssODN homology arms have significant impacts on editing efficiency; however, different alleles yield different results. For example, the study summarized above reported that optimal ssODN orientation differed depending on allele targeted, while another reported that for single-nucleotide edits, antisense orientation yielded superior editing rates [75].

In addition to ssODN, double-stranded DNA repair vectors can also be used to repair Cas9-induced DNA breaks. In one study, dsDNA template was generated to incorporate a puromycin resistance cassette and an edit to repair the F508del mutation. After puromycin selection, 6 of 36 clones were found to carry the F508del correction, giving an editing efficiency of $16.7 \%$ [57]. Of note, the puromycin resistance cassette in the donor oligo was flanked by piggyBac transposase sites, allowing researchers to excise the selection marker after successful editing.

\subsection{Viral Vector Approach to Donor DNA Delivery}

Adeno-associated virus (AAV) vectors have been used to deliver repair templates for HDR in human cells $[70,76]$. Using Cas9 RNP, researchers targeted the F508del mutation in upper airway basal stem cells (UABCs) and human bronchial epithelial cells (HBECs) from CF patients either homozygous or compound heterozygous for the F508del mutation [70]. Cells were collected, expanded, electroporated with the RNP, and then immediately transduced with an AAV6 vector carrying a repair template that included both the F508 correction and six silent mutations spanning the PAM and spacer sequence to prevent Cas9 from further editing [70]. Using this strategy, mutation correction was observed in $28 \pm 5 \%$ of F508del-homozygous UABCs, $42 \pm 15 \%$ of compound heterozygous UABCs, and $41 \pm$ $4 \%$ of homozygous HBECs [70]. Corrected cells were then expanded and seeded in air-liquid interface (ALI) culture, which promotes expansion and differentiation into a pseudostratified epithelium very similar to that of the airway in vivo. ALI cultures were then analysed for electrophysiological properties using an Ussing chamber. Corrected UABCs displayed $31 \pm$ $5 \%$ current of non-CF controls, while corrected HBECs displayed $51 \pm 3 \%$ current of non-CF 
controls [70]. Previously, it has been estimated that a 10\% increase in CFTR ion channel activity may be clinically beneficial to CF patients [77].

\section{Towards In Vivo Targeting of Cell Types in the Cystic Fibrosis Lung}

\subsection{Challenges to Editing Epithelial Cells In Vivo}

One of the major obstacles to precise genome editing is the fact that HDR does not occur in non-dividing cells $[52,56]$. The post-mitotic cells of the airway epithelium, therefore, cannot be precisely edited using HDR. Additionally, these cells turn over approximately every four months; therefore, even if editing is achieved, it must be repeated routinely, which is not possible with immunogenic vectors like AAV [78]. However, some CF mutations may be corrected by NHEJ strategies, such as the deep intronic mutation c. $3718+2477 \mathrm{C}>\mathrm{T}$, which creates a cryptic splice acceptor that results in the inclusion of a pseudoexon encoding a premature stop codon [79]. A minigene plasmid was generated containing a contiguous segment of the CFTR gene, including the relevant intron; this sequence was either c. $3718+2477 \mathrm{C}>\mathrm{T}$ mutant or WT. A second plasmid was generated encoding Cas9 and a pair of gRNAs, which targeted sites immediately upstream and downstream of the c. $3718+2477 \mathrm{C}>\mathrm{T}$ mutation in order to excise it. Plasmids were cotransfected into HEK293T cells, and minigene splicing was subsequently analysed. As measured by PCR and agarose gel densitometry, the mutation was excised in $61 \%$ of transfected cells. RT-PCR was performed on RNA isolated from transfected cells; electropherogram analysis revealed that correct splicing had been restored at $83 \%$ efficiency. Another splicing mutation, c. $3849+10 \mathrm{kbC}>\mathrm{T}$, could also potentially be treated using a similar approach.

\subsection{Towards Editing Basal Cells In Vivo}

Underneath the epithelial layer lies a population of airway basal cells, which are capable of proliferation and differentiation [80]. These multipotent stem cells represent an attractive option for targeted CRISPR, as they can be repaired by HDR and will continuously give rise to edited progeny cells. However, in order to access these basal cells in vivo, the outer epithelial layer of cells must first be stripped away. Multiple strategies have been proposed for achieving this.

Firstly, mechanical brushing of airway and/or nasal surfaces is commonly used to harvest airway epithelial cells from patients with airway diseases [81]. Scraping can clear swaths of epithelial cells while leaving basal cells undamaged [82]. Chemical stripping of the 
epithelium is difficult in vivo without risking the overall health of the airway. Previous studies of airway healing after chemical stripping of the epithelium have used chlorine gas, proteolytic enzymes, naphthalene, and irradiation [80, 83, 84]. Another potential avenue is the use of common, low-risk rhinoviruses to irritate the epithelium. Rhinovirus C and H1N1 influenza viruses, which are known to target ciliated cells in the airway, result in infected cells being shed from the epithelium [85]. Therefore, deliberate infection could potentially create access to progenitor cells, although this approach carries risk.

A less harmful approach is loosening of the tight junctions between epithelial cells to allow for leakage of CRISPR machinery into the basal layer. For example, treatment of the airways with lysophosphatidylcholine (LPC), a mild detergent, exposed the basal cells and led to significantly higher rates of lentiviral transduction than mice pretreated with phosphate-buffered saline (PBS) [86]. Mice pretreated with PBS yielded an average of $10 \pm$ $8.59 \%$ transduced cells per counted region, whereas mice pretreated with $0.1 \%$ and $0.3 \%$ LPC yielded an average of $39.7 \pm 9.2 \%$ and $24.35 \pm 8.23 \%$ respectively [86]. While the majority of transduced cells were ciliated epithelial cells, treatment with LPC did allow some instances of successful transduction in basal cells [86]. However, accurate quantification of LPC-mediated basal cell penetration would require either longer timescale experiments to evaluate presence of transduction reporter (in this case X-gal) in the next generation of surface epithelial cells post-turnover, or co-labelling with basal cell markers. Additionally, differently sized particles may permeate with different efficiency through LPC-loosened tight junctions; therefore, investigation into the effect of molecule size on permeation is also necessary to assess versatility in introducing different biochemical machinery, such as differently sized viral vectors, nanoparticle-encapsulated plasmids, CRISPR RNPs, etc.

\subsection{Transplantation of Ex Vivo-Edited Cells}

Ex vivo gene editing is an attractive option due to the fact that actively dividing progenitor cells can be edited via HDR before being differentiated into epithelial cells, and altered genomes assessed for off-target edits before being introduced into the body. The success of this approach will depend on the development of safe and efficient methods for engraftment of edited cells back into the airways and subsequent successful differentiation into the pseudostratified epithelium. Successful implantation of basal epithelial cells has been shown to be enhanced by airway pre-injury, exposing the basement membrane and creating a niche for engraftment of edited cells. NHBE cells were transduced via lentivirus to express GFP and intratracheally introduced into the airways of C57BL/6J [87]. Mice were pretreated 
with $2 \%$ polidocanol administered into the airway, a procedure previously shown to cause extensive shedding of epithelial airway cells, resulting either in exposure of basal cells or complete denuding of the basement membrane [88]. 24 hours after polidocanol treatment, GFP+ NHBE cells were administered and 48 hours later, cell retention efficiency was assessed in lung homogenates and reported as $10.48 \pm 2.86 \%$ [87].

In a similar study, $2 \%$ polidocanol was administered to mice prior to introduction of human airway basal cells (hABCs) transduced via lentivirus with luciferase. After one week, luciferase fluorescence intensity was quantified via in vivo bioluminescence imaging [89]. Mice pretreated with PBS showed no bioluminescence at any timepoint. Three of seven mice pretreated with polidocanol two hours prior to hABC cell delivery showed significant bioluminescence compared with the PBS control at both three and five weeks before dropping to insignificant levels at five and eight weeks; however, three of the ten initial hABC-treated animals died during cell delivery [89].

Another potential chemical for pretreatment is naphthalene (NA), as shown by successful implantation of a mixture of murine lung tissue stem cells (TSCs), a rare basal cell subtype, and progenitor cells [90]. TSCs cultured in vitro were found to create clones consisting of a mixture of TSCs and basal progenitor cells. Attempts to purify TSCs from these mixed pools resulted in inhibition of TSC self-renewal [90] and so transplantation was attempted with the mixed population. Similarly to the study described above, airway preinjury was found to significantly enhance cell implantation. No post-transplant repopulation occurred in NA-untreated mouse airway, nor in areas of the airway that were undamaged by NA treatment, such as the parenchyma [90]. LacZ-labelled TSC/basal progenitor cell mixtures were transplanted into the NA-injured airways of NOD/SCID/TCR- $\gamma$ mice. Two weeks post-transplant, implanted cells were found to have repopulated the injured trachea with basal and secretory cells at frequencies similar to the host-derived populations although differentiation into ciliated cells was significantly decreased compared to host populations [90].

Subsequently, transplantation was tested with GFP-marked human-derived TSC/basal progenitor cells. Two weeks post-transplantation, GFP+ cells were found in the trachea and intrapulmonary regions; as with LacZ-labelled mouse-derived cells, no successful implantation occurred in non-preinjured parenchyma [90]. In the majority of transplanted airways, transplant-derived cells were less frequent compared to host-derived cells. Host differentiation ratios were incongruent with host-derived populations, with an overrepresentation of mucus cells and underrepresentation of basal and ciliated cells [90]. 
Overall, despite representing a negligible portion of basal cells in bronchial airways, transplant-derived cells represented approximately half of epithelial secretory and ciliated cell types [90].

In another study, embryonic cell engraftment was explored as an option for repopulation of injured airway. In both human and mouse samples, tissues harvested from the canalicular stage of embryonic lung development were found to contain high concentrations of epithelial, endothelial, and mesenchymal progenitor cells [84]. Similarly to the study above, C57BL/6 mouse airways were injured by NA prior to transplantation. However, 48 hours after NA exposure, mice were also exposed to 6 Gy total body irradiation to limit endogenous host cell repopulation, which allowed transplanted cells to more successfully compete for niche engraftment [84]. Several hours after completion of this preconditioning regimen, GFP+ canalicular cells were transplanted into the airway. After 8 weeks, transplantderived cells were found to occupy an average of $11 \pm 3 \%$ of recipient lungs. After 16 weeks, transplant-derived cells occupied $28 \pm 6 \%$ of lungs [84]. Of note, upon staining for different marker proteins of lung cell types, several cells were found to express CFTR, though protein functionality and ion conductance were not assayed [84], Researchers then attempted to use the preconditioning regimen to prime NOD/SCID mouse lungs for transplant with cells derived from human embryos. However, due to the increased sensitivity to radiation in the mouse line, a lower dose was used [84]. After transplant, antibody staining revealed significantly reduced integration of human donor cells compared to mouse embryonic cell transplantation [84]. This was hypothesized to be due to the lower dose of radiation, or due to differences between human and mouse cytokines, which has previously been implicated as a hindrance to chimerism [84].

It has also been proposed that basal cells from the sinuses of CF patients could be edited ex vivo and then edited with CRISPR/Cas9 to repair the CFTR mutation; edited cells would then be engrafted back into the sinuses using a biomaterial to promote engraftment [54].

\section{Technologies Derived from the CRISPR-Cas9 System}

\subsection{Homology-Independent Targeted Integration of CFTR Superexons}

Homology-independent targeted integration (HITI) allows for targeted insertion of DNA even in non-dividing cells [91, 92]. This process utilizes the mechanisms active during NHEJ repair of DSBs, which is active at all stages of the cell cycle. The repair plasmid, instead of including homology arms, includes the same crRNA sequence as present at the 
genomic cut site; however, in the repair donor, it is encoded in the reverse orientation [91, 92]. When repair is executed in the forward orientation, insertion takes place upstream of the genomic PAM site, therefore interrupting homology to the guide RNA and preventing recutting; when repair is executed in the reverse direction, however, the crRNA sequence encoded in the template becomes re-reversed so that the Cas9 RNP can recognize, bind, and excise it $[91,92]$. Due to this process, improperly integrated repairs will repeatedly be excised until they integrate in the proper orientation $[91,92]$. Remarkably, resection of DSB ends did not appear to result in deletions or frameshift mutations in the majority of cases [92]. In vitro GFP knock-in was successful in non-dividing neurons, in which no HDR occurred [92]. In vivo editing of non-dividing mouse neurons revealed $3 \%$ absolute knock-in efficiency [92]. Overall, the use of HITI represents an opportunity to execute controlled, non-random editing in non-dividing cells. In the case of CFTR, HITI could potentially be used to insert large sections of coding sequences or even whole cDNAs, as discussed in Section 6.3.

\subsection{Adenine and Cytidine Base Editing}

Base editing approaches enable precise editing of single nucleotides, particularly cytosine to thymine $(C>T)$ [93], or adenine to guanine $(A>G)[94,95]$. Adenine base editing utilizes a fused pair of engineered enzymes: an engineered form of Cas 9 that forms singlestranded rather than double-stranded DNA breaks (commonly referred to as Cas9 nickase), and an adenosine deaminase obtained by directed evolution which is capable of hydrolysing adenine into inosine (which is interpreted by replication machinery as guanine) [94]. At present, several versions of this fusion protein exist, but the mechanism of editing is consistent $[93,95,96]$. The modified Cas9 fusion protein is guided to the target locus by a crRNA, and DNA is bound and "opened." The adenine deaminase base editor (ABE) hydrolyses adenine residues within a five-nucleotide editing window. Then, the modified Cas9 nicks the non-edited strand, which prompts the cell to repair the non-edited strand based on complementarity to the edited strand, therefore permanently incorporating the desired edit [94]. The lack of DSB formation leads to a lower occurrence of apoptosis and fewer risks of off-target DNA edits $[93,94]$. However, adenine base editors indiscriminately edit any and all adenines within the editing window, which can lead to unwanted changes if a canonical adenine is present adjacent to the mutated residue.

The ability to edit adenine to guanine represents an opportunity to transform stop codons (UGA, UAG, UAA) into codons encoding tryptophan (UGG). This is potentially invaluable for cystic fibrosis, as the current most promising CF pharmaceutical intervention 
is unable to rescue defects caused by premature stop codons, but is effective in rescuing most missense mutations (which a STOP $>$ Trp would most likely represent) [26]. Additionally, by targeting the noncoding strand, a broader range of changes can be made, as outlined in Table 2.

In a $\mathrm{CF}$ intestinal organoid biobank, encompassing mutations representing nearly $50 \%$ of the Dutch CF population, $21 \%$ of mutations were found by bioinformatic analysis to be potentially rescuable using ABEs [97]. Several such alleles were targeted for treatment by ABEs including the R785X mutation; this yielded an editing efficiency five-fold higher than that of Cas9-mediated DSB and HDR [97]. Subsequently, two more mutations (W1282X and R553X) were corrected in clonal cell lines using ABEs [97]. In this study, editing efficiency was quantified by phenotypic screening; therefore it is possible that more cells received the desired edit, but also acquired deleterious off-target mutations that prevented forskolininduced swelling (FIS) response or organoid formation in general. Analysis of off-target effects revealed no significant alterations at predicted off-target sites, nor were increases observed in the occurrence of $A>G$ changes across the entire genome [97].

The editing efficiency of ABEs has recently been increased by incorporating chemical modifications into the ABE mRNA and guide RNAs. Uridine-depleted (UD) mRNA encoding the codon-optimized ABE RA6.3 was created by silently altering codons in order to minimize the number of uridines in the transcript, while remaining uridines were replaced with 5-methoxyuridine to create 5-methoxyuridine-modified (5moU) mRNA, which is a more stable template for translation [98]. 5moU ABE mRNA yielded unexpectedly low $A>G$ editing rates when targeted to a genomic site known to have high editing rates upon delivery of RA6.3 encoded by plasmid DNA (pDNA) [98]. However, combining the 5moU ABE mRNA electroporated with 2'O-methyl 3'-phosphorothioate modified sgRNA yielded editing rates that exceeded plasmid encoded ABE RA6.3 and sgRNA at three separate genomic sites [98]. This approach was then used to target the CFTR W1282X mutation, which is caused by a $\mathrm{G}>\mathrm{A}$ mutation. Two A residues lay within the editing window, the editing target (A9G) and a non-target bystander (A5G). Modified sgRNA was electroporated alongside either unmodified or 5moU-modified ABE mRNA. Off-target editing efficiency at A5G was significantly higher with the 5moU ABE mRNA [98], although editing efficiency was higher at A9G than at A5G with both 5moU and unmodified mRNA. Base editing at A5G modifies the glutamine residue encoded by the codon immediately preceding the target codon (Q1281) to an arginine, and so the phenotypic effect of this Q1281R substitution was assessed in clones. Clones containing the corrected nonsense mutation with or without the Q1281R 
mutation exhibited normal CFTR channel activity, suggesting that ABE editing restored CFTR function and that the presence of the Q1281R mutation in the same allele did not hinder CFTR function [98].

Another base editing technology, using cytidine deaminases, is capable of converting cytosine nucleotides into thymines [93]. There are 147 known $\mathrm{C}>\mathrm{T}$ point mutations listed in the Cystic Fibrosis Mutations Database which could potentially be addressed by this technology [99]. Similar to adenine base editors, cytosine base editors edit all cytosine residues within the editing window, which can lead to unwanted non-target mutations.

\subsection{Prime Editing}

Prime editing represents an opportunity to pair the advantages of single-stranded DNA cuts (as explored in base editing) with the expanded precision editing capabilities of homology-directed repair. However, rather than chemical modification of individual nucleotides (as in the case of base editing), the prime editing approach rebuilds damaged DNA by using an engineered reverse transcriptase and an RNA repair template [100]. The reverse transcriptase is fused with a prime editing gRNA (pegRNA), which contains an RNA template for the desired edits. Similar to base editing, after editing has occurred, the nonedited strand is nicked and subsequently repaired according to complementarity to the edited strand, thus perpetuating the desired changes [100]. This allows editing on a larger scale than the single-nucleotide edits of base editing and eludes the issue of base editors indiscriminately editing all $\mathrm{A}$ or $\mathrm{C}$ nucleotides within the editing window. As in the case of base editing, the lack of double-strand break formation leads to lower rates of apoptosis and off-target DNA damage [93, 94, 100].

However, prime editing requires a large fusion protein $(6.3 \mathrm{~kb}$ cDNA and $230 \mathrm{kDa}$, compared with $4 \mathrm{~kb}$ cDNA and $160 \mathrm{kDa}$ protein SpCas9), which could lead to difficulties in efficient delivery [100]. Much more research is required in the optimization of delivery, as well as optimization of pegRNA design and potential chemical modifications, which have been shown to greatly enhance Cas9-associated guide RNAs [64-67].

\section{Non-Cas9 Approaches to Gene Editing of CFTR}

\subsection{Transcription-Activator-Like Effector Nucleases (TALENs)}

Additionally, non-CRISPR-Cas9 gene editing therapies have recently been explored. TALEN-mediated homologous recombination was recently used to correct the F508del mutation in iPSCs, which were then expanded into intestinal organoids [101]. A TALEN 
plasmid was nucleofected alongside a donor vector carrying the desired mutation correction as well as a puromycin resistance selection cassette flanked by PiggyBac transposon sites; after correction and selection, the selection cassette was removed via the PiggyBac system [101]. Corrected iPSCs showed CFTR protein abundance and localization comparable to nonCF cells; corrected and uncorrected iPSCs were then expanded into intestinal organoids [101]. CFTR function was assayed using FIS assay; upon exposure to forskolin, corrected organoids increased their surface area to $177 \%$, while uncorrected organoids increased to $103 \%$, with $100 \%$ representing the pretreatment surface area [101]. Additionally, corrected organoids were found to be responsive to the clinically approved double-combination drug VX-770/VX-809 (marketed as Orkambi), suggesting that DNA editing-based therapies can be combined with pharmaceutical intervention to enhance clinical outcome [101].

\subsection{AsCas12a/Cpf1}

The Cas protein AsCas12a (also commonly referred to as $\mathrm{Cpf1}$ ), derived from Acidaminococcus, recognizes the PAM sequence TTTV. This nuclease has recently been used in lieu of Cas9 in order to excise intronic splice altering CFTR mutations. Due to AsCas12a's tendency to create deletions $>4 \mathrm{bp}$, a single guide rather than a pair was used to target the desired mutation; this is potentially advantageous because fewer DSBs leads to fewer opportunities for apoptosis induction and off-target indel formation. The mutations $3272-26 \mathrm{~A}>\mathrm{G}$ and $3849+10 \mathrm{kbC}>\mathrm{T}$ CFTR were targeted using AsCas12a in first a minigene model and then in primary airway cells and intestinal organoids. In primary airway cells derived from a 3272-26A $>$ G/F508del compound heterozygote, lentiviral delivery of AsCas $12 \mathrm{a}$ with a single guide yielded $30 \%$ cutting efficiency and 13 -fold rescue of aberrant splicing without puromycin selection [102]. In human intestinal organoids derived from a 3272-26A $>$ G/4218ins T compound heterozygote, lentiviral delivery of AsCas12a with a single guide resulted in restoration of proper splicing from $69 \%$ to $95 \%$ [102]. FIS response in edited organoids increased 2.8-fold compared to unedited organoids, similar to the result obtained by rescue using lentiviral introduction of non-mutant CFTR cDNA [102]. These protocols were repeated with a guide targeting the $3849+10 \mathrm{kbC}>\mathrm{T}$ allele; in compound heterozygous $3849+10 \mathrm{kbC}>\mathrm{T} / \mathrm{F} 508 \mathrm{del}$ primary airway cells, a 20\% indel rate was achieved; in intestinal organoids with the same genotype, a 30\% indel rate was achieved, and FIS swelling was restored to the same level as that achieved by lentiviral introduction of nonmutant CFTR cDNA [102]. A pair of guide RNAs accompanied by SpCas9, also introduced via lentivirus, yielded significantly lower rates of FIS responsiveness than both AsCas12a- 
corrected organoids and WT CFTR cDNA-expressing organoids [102]. However, long-term expression of Cas9 from lentiviral integration raises safety concerns as it has been shown to exacerbate off-target DSBs throughout the genome [61], and so comparison with more clinically viable delivery strategies is necessary in the future.

\subsection{Zinc Finger Nucleases (ZFNs)}

Integration of a CFTR superexon (as discussed in Section 5.1), encoding exons 11-27, has already been studied using zinc finger nucleases (ZFNs) [103]. The superexon and plasmids encoding ZFNs were transfected into an F508del-homozygous cell line (CFBE41o-) [103]. The superexon was integrated at the target locus (just upstream of the F508 locus) with a targeting efficiency of approximately $10 \%$ [103]. Monoallelic integration was found in five out of 48 sequenced clones; three of these five clones produced chimeric CFTR mRNA as measured by allele-specific RT-PCR after drug-induced demethylation of the CFTR locus [103]. One successfully edited clonal cell line was then expanded into a pseudostratified epithelium in ALI culture, and subsequently analysed using an Ussing chamber [103]. While unedited CFBE41o- cells yielded no significant transepithelial current, edited cells displayed current comparable to wild-type airway epithelial cells [103].

ZFNs have also been used to correct CFTR defects in CF patient-derived airway basal cells of various genotypes. Firstly, ZFN mRNA targeting the F508del mutation was electroporated alongside a 200-bp ssODN donor molecule consisting of the restoring edit flanked by $100 \mathrm{bp}$ of homology [104]. Electroporation of the targeting ZFN mRNA alone yielded $44.6 \pm 2.4 \%$ indel formation while codelivery with the repair oligo yielded $10.6 \pm$ $2.6 \%$ correction of the F508del mutation (measured by percent of sequenced alleles harbouring each result), yielding an HDR:indel ratio of 1:5 [104]. In order to increase rates of HDR, a longer DNA donor containing two $1 \mathrm{~kb}$ homology arms was introduced via AAV6 transduction. This approach increased the editing efficiency to $31.0 \pm 4.0 \%$ and an improved HDR:indel ratio of 3:2 [104]. Cells corrected using the AAV6 donor strategy were expanded on ALI culture and assayed using an Ussing chamber, along with non-CF cells and F508delhomozygous cells treated with the CF double-combination drug VX-809/VX-770. When exposed to the CFTR activator forskolin, corrected cells exhibited $40.2 \%$ of non-CF increase in current, compared with $26.7 \%$ of the normal CFTR current in CF F508del cells which had been treated with VX-809/VX-770- [104]. When exposed to the CFTR inhibitor CFTRinh172 , corrected cells exhibited $49.4 \%$ of non-CF reduction in current, compared with $31.3 \%$ non-CF current reduction exhibited by VX-809/VX-770-treated cells [104]. 
Secondly, ZFN mRNA targeting intron 8 was electroporated followed by AAV6 transduction of the $C F T R$ superexon cassette $S A-C F T R_{9-27}-p A$. This construct encodes wildtype CFTR exons 9-27; provided that patient mutations occur in or after exon 9, targeted addition of this superexon in intron 8 will create a functional CFTR gene. Electroporation of the targeting ZFN mRNA alone yielded $86.5 \%$ indel formation; codelivery with AAV6 donor yielded $56.5 \pm 7.4 \%$ of NGS-surveyed alleles carrying the successfully integrated superexon, and an integration:indel ratio of 5:3 [104]. In two independent experiments, Ussing analysis was performed using the same comparison populations (non-CF and F508del-homozygous drug-treated) as in the prior correction strategy. Across the two experiments, there was some variation in the non-CF and superexon-integrated cells' responses to forskolin and CFTRinh172. However, in both experiments, superexon-integrated cells either matched or outperformed VX-809/VX-770-treated CF cells, as measured by the percent of non-CF current change achieved in response to both inhibition and activation. Finally, the AAV6 superexon integration approach was used in G542X/R785X compound heterozygous primary airway basal cells for an integration rate of $61.8 \% \pm 6.0 \%$ and an integration:indel ratio of $1: 2$ [104]. Corrected cells were expanded on ALI and analysed via Ussing chamber using the same comparison populations as above. When exposed to forskolin, superexon-integrated cells exhibited $33.8 \%$ of non-CF increase in current; when exposed to CFTRinh-172, superexon-integrated cells exhibited $30.6 \%$ of non-CF reduction in current; CF cells treated with VX-809/VX-770 did not respond to the CFTR activator or inhibitor [104].

\section{Conclusion}

Cystic fibrosis is on its surface an excellent candidate for a genetic engineering-based treatment approach. Despite this, genetic medicines for CF have for decades faltered in clinical trials and been deemed unsuitable for human patients. However, in the past five years, the development of CRISPR-Cas9 as a highly efficient and programmable genome editing tool has opened up a new opportunity to progress the field of CF treatment. CRISPRCas9 genome editing, and the technologies therefrom derived, represents an invaluable opportunity to correct the underlying cause of CF. In addition to the techniques discussed in this review, other alterations to various aspects of the CRISPR system have also been explored. A multitude of biochemical or structural modifications to gRNAs [64-67, 105], use of a "double nickase" approach [106], and various permutations of size and symmetry in repair template homology arms [75] have all been explored. 


\section{Expert Opinion}

The equilibrium of precision, efficiency, and overall risk to cell health are the major players in the delicate balancing act of safety and efficacy in treatment development. There is not yet a "one size fits all" approach to treatment of CF via gene editing. Different mutations demand unique approaches and targeting different cell types demand different delivery strategies.

On the molecular level, various modifications of the CRISPR system have been developed that introduce new pros and cons to the delicate balance of genome engineering. Base editing and prime editing eliminate the issue of double-stranded DNA damage but reduce the capability for large-scale edits. NHEJ-based repair strategies such as HITI allow for targeted insertion of genetic material into the genomes of nondividing cells, but precision of integration is somewhat reduced compared to homology-directed repair. Researchers must consider the respective advantages and disadvantages of each system when planning to target different $\mathrm{CF}$-causing mutations. $\mathrm{A}>\mathrm{G}$ vs. $\mathrm{C}>\mathrm{T}$ mutations, insertions vs. deletions, and intronic vs. exonic mutations demand different approaches in order to optimize treatment strategies. Editing efficiencies of selected CRISPR-based approaches outlined in this paper can be found in Table 1; however, due to differences in target mutation and experimental design, reported efficiencies should not be directly compared.

On a cellular level, one of the greatest obstacles to treating cystic fibrosis with CRISPR-Cas9 is the fact that airway epithelial cells are non-dividing, and therefore cannot be targeted with strategies that rely on HDR. Most common CF-causing mutations occur in exons, and therefore precise repair is essential, making NHEJ-based approaches unfavourable. Removal of the epithelial layer in order to target basal cells gives the opportunity for more exact repair via HDR. However, as discussed in Section 4.2, stripping of the epithelium represents a risk to the overall health of the airway. In several cases, this has been addressed by use of ex vivo approaches, as outlined in Section 4.3. Patient cells are reprogrammed into iPSCs, edited using HDR-based strategies, and subsequently expanded into cell populations capable of differentiation into a functional pseudostratified airway epithelium. An ex-vivo approach also allows for use of electroporation of RNPs, which has shown high efficacy in iPSCs derived from CF patients. However, reintroduction of edited cells into the airway remains a challenge. Just as in the case of targeting basal cells in vivo, removal of the airway epithelium for engraftment purposes can present a risk to airway health. Alternatively, it has recently been shown that edited stem cells can be grown on an 
FDA-approved surgical matrix for reimplantation into the airway [70]. While an exciting development, this approach still calls for an invasive procedure to be performed on a tissue already at risk of chronic inflammation due to the nature of $\mathrm{CF}$.

Another approach is to entirely bypass the need for HDR. Techniques such as HITI and prime editing do not depend on pathways only active during cell division. Therefore, these techniques can be used to either insert superexons of wild-type CFTR cDNA (HITI) or correct individual small-scale mutations (base and prime editing). However, techniques for delivery of such materials remains an issue. Immunogenic vectors are unfavourable due to the fact that epithelial cell turnover creates need for repeated administration. Lipid-based nanoparticles represent an opportunity to coat immunogenic agents in a less toxic packaging. However, research on packaging materials for HITI or prime editing has not yet been extensively explored.

In all gene editing approaches, the risk of off-target genome alteration remains a large concern. However, multiple recent approaches have already begun to reduce these risks. Use of technologies that do not induce double-stranded breaks, such as base or prime editing, have shown decreased off-target effects; additionally, specific engineering of protein and RNA components have been shown to further reduce off-target DNA binding [61, 93-95, 98, $100]$.

Overall, the most difficult choice facing CFTR treatment development is which cell type to target, as the different tissue layers of the pseudostratified epithelium carry unique demands and challenges. Targeting individual mutations in vivo and ex vivo may fall out of favour due to such challenges, and a true "one size fits all" (or, more realistically, "one size fits most") cure will more likely be based on superexon integration or safe-harbour insertion of CFTR cDNA. Additionally, avoiding the potential pitfalls of gene editing (off-target editing and DSB-induced apoptosis) may be avoided by correcting cells ex vivo and reimplanting them into the epithelium; however, cellular engraftment presents its own set of challenges and risks to patient health. The CRISPR system has opened the door for precise and efficient genome editing, and further adaptations of CRISPR-Cas9 have already begun leading to exciting developments in safer and more widely applicable technologies.

\section{Funding}

This paper is not funded. 


\section{Declaration of Interests}

The authors are affiliated with the University College London Institute of Child Health. S. Hart has equity in Ryboquin Ltd, a company involved in gene therapy. S. Hart is funded by the UK research charities, the Cystic Fibrosis Trust and Action Medical Research and Great Ormond Street Hospital Biomedical Research Centre. C. Graham is supported by UCL PhD studentships. The authors have no other relevant affiliations or financial involvement with any organization or entity with a financial interest in or financial conflict with the subject matter or materials discussed in the manuscript apart from those disclosed.

\section{Reviewer Disclosures}

Peer reviewers on this manuscript have no relevant financial relationships or otherwise to disclose.

\section{References}

1. Elborn, J.S., Cystic fibrosis. The Lancet, 2016. 388(10059): p. 2519-2531.

2. Ratjen, F., et al., Cystic fibrosis. Nat Rev Dis Primers, 2015. 1: p. 15010.

3. Keogh, R.H., et al., Up-to-date and projected estimates of survival for people with cystic fibrosis using baseline characteristics: A longitudinal study using UK patient registry data. J Cyst Fibros, 2018. 17(2): p. 218-227.

4. Hinds, D.M., et al., Cystic fibrosis in El Salvador. Pediatr Pulmonol, 2019. 54(4): p. 369-371.

5. Matsui, H., et al., Evidence for Periciliary Liquid Layer Depletion, Not Abnormal Ion Composition, in the Pathogenesis of Cystic Fibrosis Airways Disease. Cell, 1998. 95(7): p. 1005-1015.

6. Rafeeq, M.M. and H.A.S. Murad, Cystic fibrosis: current therapeutic targets and future approaches. J Transl Med, 2017. 15(1): p. 84.

7. Belkin, R.A., et al., Risk factors for death of patients with cystic fibrosis awaiting lung transplantation. Am J Respir Crit Care Med, 2006. 173(6): p. 659-66.

8. Bhagirath, A.Y., et al., Cystic fibrosis lung environment and Pseudomonas aeruginosa infection. BMC Pulm Med, 2016. 16(1): p. 174.

9. Marcos, V., et al., Free DNA in cystic fibrosis airway fluids correlates with airflow obstruction. Mediators Inflamm, 2015. 2015: p. 408935.

10. Treacy, K., et al., Mucociliary clearance in cystic fibrosis: physiology and pharmacological treatments. Paediatrics and Child Health, 2011. 21(9): p. 425-430.

11. Pezzulo, A.A., et al., Reduced airway surface $\mathrm{pH}$ impairs bacterial killing in the porcine cystic fibrosis lung. Nature, 2012. 487(7405): p. 109-13.

12. Abou Alaiwa, M.H., et al., Neonates with cystic fibrosis have a reduced nasal liquid $\mathrm{pH}$; a small pilot study. J Cyst Fibros, 2014. 13(4): p. 373-7.

13. Coakley, R., et al., Abnormal surface liquid pH regulation by cultured cystic fibrosis bronchial epithelium. Proceedings of the National Academy of Science. 100.

14. Tang, X.X., et al., Acidic $p H$ increases airway surface liquid viscosity in cystic fibrosis. J Clin Invest, 2016. 126(3): p. 879-91.

15. Schultz, A., et al., Airway surface liquid $\mathrm{pH}$ is not acidic in children with cystic fibrosis. Nat Commun, 2017. 8(1): p. 1409. 
16. Abou Alaiwa, M.H., et al., $\mathrm{pH}$ modulates the activity and synergism of the airway surface liquid antimicrobials beta-defensin-3 and LL-37. Proc Natl Acad Sci U S A, 2014. 111(52): p. 18703-8.

17. Benedetto, R., et al., Transport properties in CFTR-/- knockout piglets suggest normal airway surface liquid $\mathrm{pH}$ and enhanced amiloride-sensitive $\mathrm{Na}(+)$ absorption. Pflugers Arch, 2020.

18. Brockbank, S., et al., Effect of cystic fibrosis exacerbations on neutrophil function. Int Immunopharmacol, 2005. 5(3): p. 601-8.

19. Downey, D.G., S.C. Bell, and J.S. Elborn, Neutrophils in cystic fibrosis. Thorax, 2009. 64(1): p. 81-8.

20. Gifford, A.M. and J.D. Chalmers, The role of neutrophils in cystic fibrosis. Curr Opin Hematol, 2014. 21(1): p. 16-22.

21. Prayle, A. and A.R. Smyth, Aminoglycoside use in cystic fibrosis: therapeutic strategies and toxicity. Curr Opin Pulm Med, 2010. 16(6): p. 604-10.

22. Castellani, $\mathrm{C}$., et al., Consensus on the use and interpretation of cystic fibrosis mutation analysis in clinical practice. J Cyst Fibros, 2008. 7(3): p. 179-96.

23. Cutting, G.R., CFTR2. 2011.

24. De Boeck, K., et al., The relative frequency of CFTR mutation classes in European patients with cystic fibrosis. J Cyst Fibros, 2014. 13(4): p. 403-9.

25. Veit, G., et al., From CFTR biology toward combinatorial pharmacotherapy: expanded classification of cystic fibrosis mutations. Mol Biol Cell, 2016. 27(3): p. 424-33.

26. Bear, C.E., A Therapy for Most with Cystic Fibrosis. Cell, 2020. 180(2): p. 211.

27. Keating, D., et al., VX-445-Tezacaftor-Ivacaftor in Patients with Cystic Fibrosis and One or Two Phe508del Alleles. N Engl J Med, 2018. 379(17): p. 1612-1620.

28. Middleton, P.G., et al., Elexacaftor-Tezacaftor-Ivacaftor for Cystic Fibrosis with a Single Phe508del Allele. N Engl J Med, 2019. 381(19): p. 1809-1819.

29. McHugh, D.R., et al., A G542X cystic fibrosis mouse model for examining nonsense mutation directed therapies. PLoS One, 2018. 13(6): p. e0199573.

30. Davies, G., et al., Characterising burden of treatment in cystic fibrosis to identify priority areas for clinical trials. J Cyst Fibros, 2019.

31. Nichols, D. Impact of Discontinuing Chronic Therapies in People With Cystic Fibrosis on Highly Effective CFTR Modulator Therapy (SIMPLIFY). 202019 October 2020; Available from: https://clinicaltrials.gov/ct2/show/NCT04378153.

32. Trikafta Approval: Community Frequently Asked Questions. 2020; Available from: https://www.cff.org/Life-With-CF/Treatments-and-Therapies/Trikafta-Approval-CommunityFAQ/.

33. Schneider-Futschik, E.K., Beyond cystic fibrosis transmembrane conductance regulator therapy: a perspective on gene therapy and small molecule treatment for cystic fibrosis. Gene Ther, 2019. 26(9): p. 354-362.

34. Griesenbach, U. and E.W. Alton, Moving forward: cystic fibrosis gene therapy. Hum Mol Genet, 2013. 22(R1): p. R52-8.

35. Carneiro, A., et al., Novel Lung Tropic Adeno-Associated Virus Capsids for Therapeutic Gene Delivery. Hum Gene Ther, 2020. 31(17-18): p. 996-1009.

36. Alton, E.W., et al., Preparation for a first-in-man lentivirus trial in patients with cystic fibrosis. Thorax, 2017. 72(2): p. 137-147.

37. Alton, E.W.F.W., et al., Repeated nebulisation of non-viral CFTR gene therapy in patients with cystic fibrosis: a randomised, double-blind, placebo-controlled, phase $2 b$ trial. The Lancet Respiratory Medicine, 2015. 3(9): p. 684-691.

38. Mali, P., et al., RNA-guided human genome engineering via Cas9. Science, 2013. 339(6121): p. 823-6.

39. Jinek, M., et al., A programmable dual-RNA-guided DNA endonuclease in adaptive bacterial immunity. Science, 2012. 
40. Barrangou, R., et al., CRISPR provides acquired resistance against viruses in prokaryotes. Science, 2007. 315: p. 1709-1712.

41. Mojica, F.J.M. and L. Montoliu, On the Origin of CRISPR-Cas Technology: From Prokaryotes to Mammals. Trends Microbiol, 2016. 24(10): p. 811-820.

42. Brouns, S., et al., Small CRISPR RNAs Guide Antiviral Defense in Prokaryotes. Science, 2008. 321: p. 960-963.

43. Mojica, F.J.M., et al., Short motif sequences determine the targets of the prokaryotic CRISPR defence system. Microbiology, 2009. 155(Pt 3): p. 733-740.

44. Cong, L., et al., Multiplex genome engineering using CRISPR/Cas systems. Science, 2013. 339: p. 819-823.

45. Canver, M.C., et al., Characterization of genomic deletion efficiency mediated by clustered regularly interspaced short palindromic repeats (CRISPR)/Cas9 nuclease system in mammalian cells. J Biol Chem, 2014. 289(31): p. 21312-24.

46. Lieber, M., The mechanism of human nonhomologous DNA end joining. J Biol Chem, 2007. 283.

47. Burma, S., B.P. Chen, and D.J. Chen, Role of non-homologous end joining (NHEJ) in maintaining genomic integrity. DNA Repair (Amst), 2006. 5(9-10): p. 1042-8.

48. Davis, A.J. and D.J. Chen, DNA double strand break repair via non-homologous end-joining. Transl Cancer Res, 2013. 2(3): p. 130-143.

49. van Gent, D., J. Hoeijmakers, and R. Kanaar, Chromosomal stability and the DNA doublestranded break connection. Nat Rev Genet, 2001. 2: p. 196-206.

50. Lees-Miller, S.P. and K. Meek, Repair of DNA double strand breaks by non-homologous end joining. Biochimie, 2003. 85(11): p. 1161-73.

51. Liang, F., et al., Homology-directed repair is a major double-strand break repair pathway in mammalian cells. Proceedings of the National Academy of Science, 1998. 95: p. 5172-5177.

52. Brandsma, I. and D.C. Gent, Pathway choice in DNA double strand break repair: observations of a balancing act. Genome Integr, 2012. 3(1): p. 9.

53. Hollywood, J.A., et al., Analysis of gene repair tracts from Cas9/gRNA double-stranded breaks in the human CFTR gene. Sci Rep, 2016. 6: p. 32230.

54. Kosicki, M., K. Tomberg, and A. Bradley, Repair of double-strand breaks induced by CRISPRCas9 leads to large deletions and complex rearrangements. Nat Biotechnol, 2018. 36(8): p. 765-771.

55. Chu, V.T., et al., Increasing the efficiency of homology-directed repair for CRISPR-Cas9induced precise gene editing in mammalian cells. Nat Biotechnol, 2015. 33(5): p. 543-8.

56. Mao, Z., et al., Comparison of nonhomologous end joining and homologous recombination in human cells. DNA Repair (Amst), 2008. 7(10): p. 1765-71.

57. Firth, A.L., et al., Functional Gene Correction for Cystic Fibrosis in Lung Epithelial Cells Generated from Patient iPSCs. Cell Rep, 2015. 12(9): p. 1385-90.

58. Schwank, G., et al., Functional repair of CFTR by CRISPR/Cas9 in intestinal stem cell organoids of cystic fibrosis patients. Cell Stem Cell, 2013. 13(6): p. 653-8.

59. Liang, X., et al., Rapid and highly efficient mammalian cell engineering via Cas9 protein transfection. J Biotechnol, 2015. 208: p. 44-53.

60. Kim, S., et al., Highly efficient RNA-guided genome editing in human cells via delivery of purified Cas9 ribonucleoproteins. Genome Res, 2014. 24(6): p. 1012-9.

61. Chen, Y., et al., A Self-restricted CRISPR System to Reduce Off-target Effects. Mol Ther, 2016. 24(9): p. 1508-10.

62. Mignon, C., R. Sodoyer, and B. Werle, Antibiotic-free selection in biotherapeutics: now and forever. Pathogens, 2015. 4(2): p. 157-81.

63. Liu, J., et al., Fast and Efficient CRISPR/Cas9 Genome Editing In Vivo Enabled by Bioreducible Lipid and Messenger RNA Nanoparticles. Adv Mater, 2019. 31(33): p. e1902575. 
64. Ryan, D.E., et al., Improving CRISPR-Cas specificity with chemical modifications in singleguide RNAs. Nucleic Acids Res, 2018. 46(2): p. 792-803.

65. Cromwell, C.R., et al., Incorporation of bridged nucleic acids into CRISPR RNAs improves Cas9 endonuclease specificity. Nat Commun, 2018. 9(1): p. 1448.

66. Yin, H., et al., Partial DNA-guided Cas9 enables genome editing with reduced off-target activity. Nat Chem Biol, 2018. 14(3): p. 311-316.

67. Basila, M., M.L. Kelley, and A.V.B. Smith, Minimal 2'-O-methyl phosphorothioate linkage modification pattern of synthetic guide RNAs for increased stability and efficient CRISPR-Cas9 gene editing avoiding cellular toxicity. PLoS One, 2017. 12(11): p. e0188593.

68. Wilbie, D., J. Walther, and E. Mastrobattista, Delivery Aspects of CRISPR/Cas for in Vivo Genome Editing. Acc Chem Res, 2019. 52(6): p. 1555-1564.

69. Ruan, J., et al., Efficient Gene Editing at Major CFTR Mutation Loci. Mol Ther Nucleic Acids, 2019. 16: p. 73-81.

70. Vaidyanathan, S., et al., Highly efficient repair of the $\Delta F 508$ mutation in airway stem cells of cystic fibrosis patients with functional rescue of the differentiated epithelia. BioRxiv, 2019.

71. Cheng, Q., et al., Selective organ targeting (SORT) nanoparticles for tissue-specific mRNA delivery and CRISPR-Cas gene editing. Nat Nanotechnol, 2020. 15: p. 313-320.

72. Miller, J.B., et al., Non-Viral CRISPR/Cas Gene Editing In Vitro and In Vivo Enabled by Synthetic Nanoparticle Co-Delivery of Cas9 mRNA and sgRNA. Angew Chem Int Ed Engl, 2017. 56(4): p. 1059-1063.

73. Wei, T., et al., Systemic nanoparticle delivery of CRISPR-Cas9 ribonucleoproteins for effective tissue specific genome editing. Nat Commun, 2020.11(1): p. 3232.

74. Manunta, M.D.I., et al., Delivery of ENaC siRNA to epithelial cells mediated by a targeted nanocomplex: a therapeutic strategy for cystic fibrosis. Sci Rep, 2017. 7(1): p. 700.

75. Okamoto, S., et al., Highly efficient genome editing for single-base substitutions using optimized ssODNs with Cas9-RNPs. Sci Rep, 2019. 9(1): p. 4811.

76. Martin, R.M., et al., Highly Efficient and Marker-free Genome Editing of Human Pluripotent Stem Cells by CRISPR-Cas9 RNP and AAV6 Donor-Mediated Homologous Recombination. Cell Stem Cell, 2019. 24(5): p. 821-828 e5.

77. Cutting, G.R., Cystic fibrosis genetics: from molecular understanding to clinical application. Nat Rev Genet, 2015. 16(1): p. 45-56.

78. Verdera, H.C., K. Kuranda, and F. Mingozzi, AAV Vector Immunogenicity in Humans: A Long Journey to Successful Gene Transfer. Mol Ther, 2020. 28(3): p. 723-746.

79. Sanz, D.J., et al., Cas9/gRNA targeted excision of cystic fibrosis-causing deep-intronic splicing mutations restores normal splicing of CFTR mRNA. PLoS One, 2017. 12(9): p. e0184009.

80. Tuck, S.A., et al., Time course of airway remodelling after an acute chlorine gas exposure in mice. Respir Res, 2008. 9: p. 61.

81. Ghosh, B., et al., Strong correlation between air-liquid interface cultures and in vivo transcriptomics of nasal brush biopsy. Am J Physiol Lung Cell Mol Physiol, 2020.

82. Erjefiilt, J.S., et al., In vivo restitution of airway epithelium. Cell and Tissue Research, 1995.

83. Machado, M.N., et al., Papain-induced experimental pulmonary emphysema in male and female mice. Respir Physiol Neurobiol, 2014. 200: p. 90-6.

84. Rosen, C., et al., Preconditioning allows engraftment of mouse and human embryonic lung cells, enabling lung repair in mice. Nat Med, 2015. 21(8): p. 869-79.

85. Griggs, T.F., et al., Rhinovirus C targets ciliated airway epithelial cells. Respir Res, 2017. 18(1): p. 84.

86. Cmielewski, P., et al., Gene therapy for Cystic Fibrosis: Improved delivery techniques and conditioning with lysophosphatidylcholine enhance lentiviral gene transfer in mouse lung airways. Exp Lung Res, 2017. 43(9-10): p. 426-433.

87. Gui, L., et al., Efficient intratracheal delivery of airway epithelial cells in mice and pigs. Am J Physiol Lung Cell Mol Physiol, 2015. 308(2): p. L221-8. 
88. Leblond, A., et al., Developing cell therapy techniques for respiratory disease: intratracheal delivery of genetically engineered stem cells in a murine model of airway injury. Hum Gen Ther, 2009. 20(11).

89. Farrow, N., et al., Epithelial disruption: a new paradigm enabling human airway stem cell transplantation. Stem Cell Res Ther, 2018. 9(1): p. 153.

90. Ghosh, M., et al., Transplantation of Airway Epithelial Stem/Progenitor Cells: A Future for Cell-Based Therapy. Am J Respir Cell Mol Biol, 2017. 56(1): p. 1-10.

91. Suzuki, K. and J.C. Izpisua Belmonte, In vivo genome editing via the HITI method as a tool for gene therapy. J Hum Genet, 2018. 63(2): p. 157-164.

92. Suzuki, K., et al., In vivo genome editing via CRISPR/Cas9 mediated homology-independent targeted integration. Nature, 2016. 540(7631): p. 144-149.

93. Komor, A.C., et al., Programmable editing of a target base in genomic DNA without doublestranded DNA cleavage. Nature, 2016. 533(7603): p. 420-4.

94. Gaudelli, N.M., et al., Programmable base editing of $A^{*} T$ to $G^{*} C$ in genomic DNA without DNA cleavage. Nature, 2017. 551(7681): p. 464-471.

95. Komor, A.C., A.H. Badran, and D.R. Liu, Editing the Genome Without Double-Stranded DNA Breaks. ACS Chem Biol, 2018. 13(2): p. 383-388.

96. Grunewald, J., et al., A dual-deaminase CRISPR base editor enables concurrent adenine and cytosine editing. Nat Biotechnol, 2020. 38(7): p. 861-864.

97. Geurts, M.H., et al., CRISPR-Based Adenine Editors Correct Nonsense Mutations in a Cystic Fibrosis Organoid Biobank. Cell Stem Cell, 2020.

98. Jiang, T., et al., Chemical modifications of adenine base editor MRNA and guide RNA expand its application scope. Nat Commun, 2020. 11(1): p. 1979.

99. Cystic Fibrosis Mutation Database. 2011; Available from: http://www.genet.sickkids.on.ca/.

100. Anzalone, A.V., et al., Search-and-replace genome editing without double-strand breaks or donor DNA. Nature, 2019. 576(7785): p. 149-157.

101. Fleischer, A., et al., iPSC-Derived Intestinal Organoids from Cystic Fibrosis Patients Acquire CFTR Activity upon TALEN-Mediated Repair of the p.F508del Mutation. Mol Ther Methods Clin Dev, 2020. 17: p. 858-870.

102. Maule, G., et al., Allele specific repair of splicing mutations in cystic fibrosis through AsCas12a genome editing. Nat Commun, 2019. 10(1): p. 3556.

103. Bednarski, C., et al., Targeted Integration of a Super-Exon into the CFTR Locus Leads to Functional Correction of a Cystic Fibrosis Cell Line Model. PLoS One, 2016. 11(8): p. e0161072.

104. Suzuki, S., et al., Highly Efficient Gene Editing of Cystic Fibrosis Patient-Derived Airway Basal Cells Results in Functional CFTR Correction. Mol Ther, 2020. 28(7): p. 1684-1695.

105. Fu, Y., et al., Improving CRISPR-Cas nuclease specificity using truncated guide RNAs. Nat Biotechnol, 2014. 32(3): p. 279-284.

106. Ran, F.A., et al., Double nicking by RNA-guided CRISPR Cas9 for enhanced genome editing specificity. Cell, 2013. 154(6): p. 1380-9. 


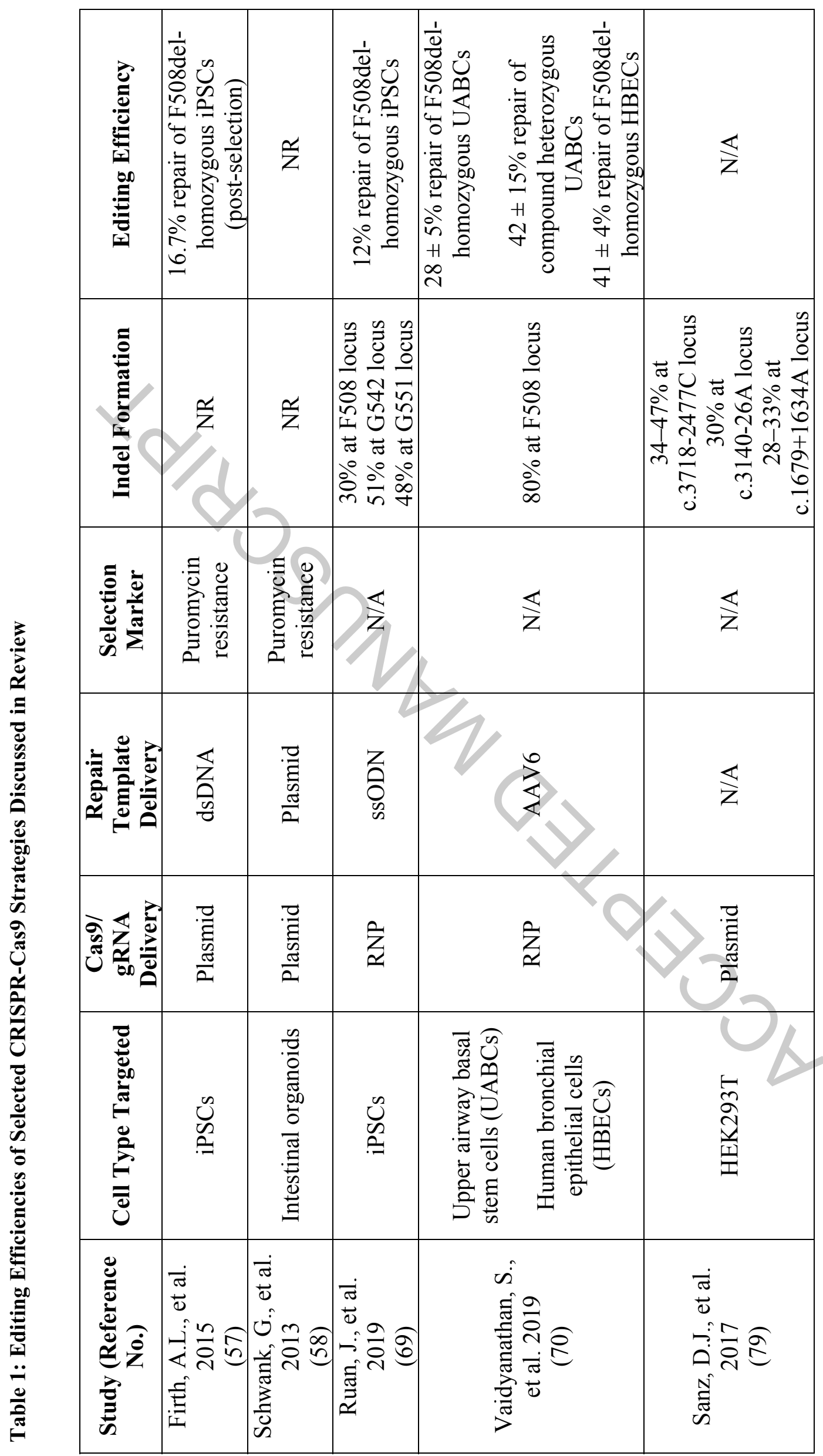




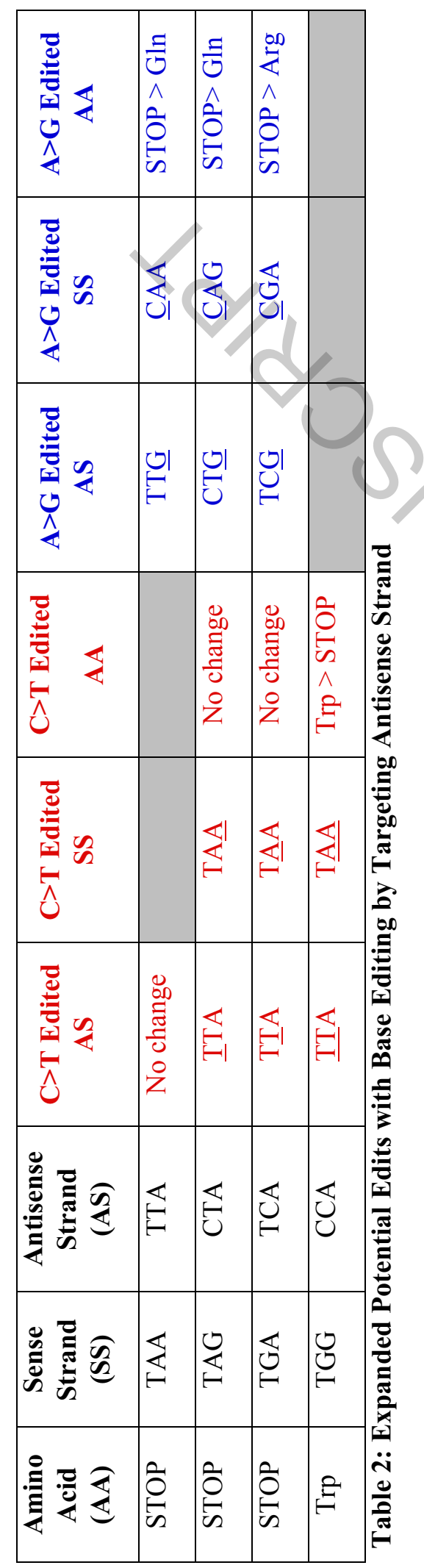




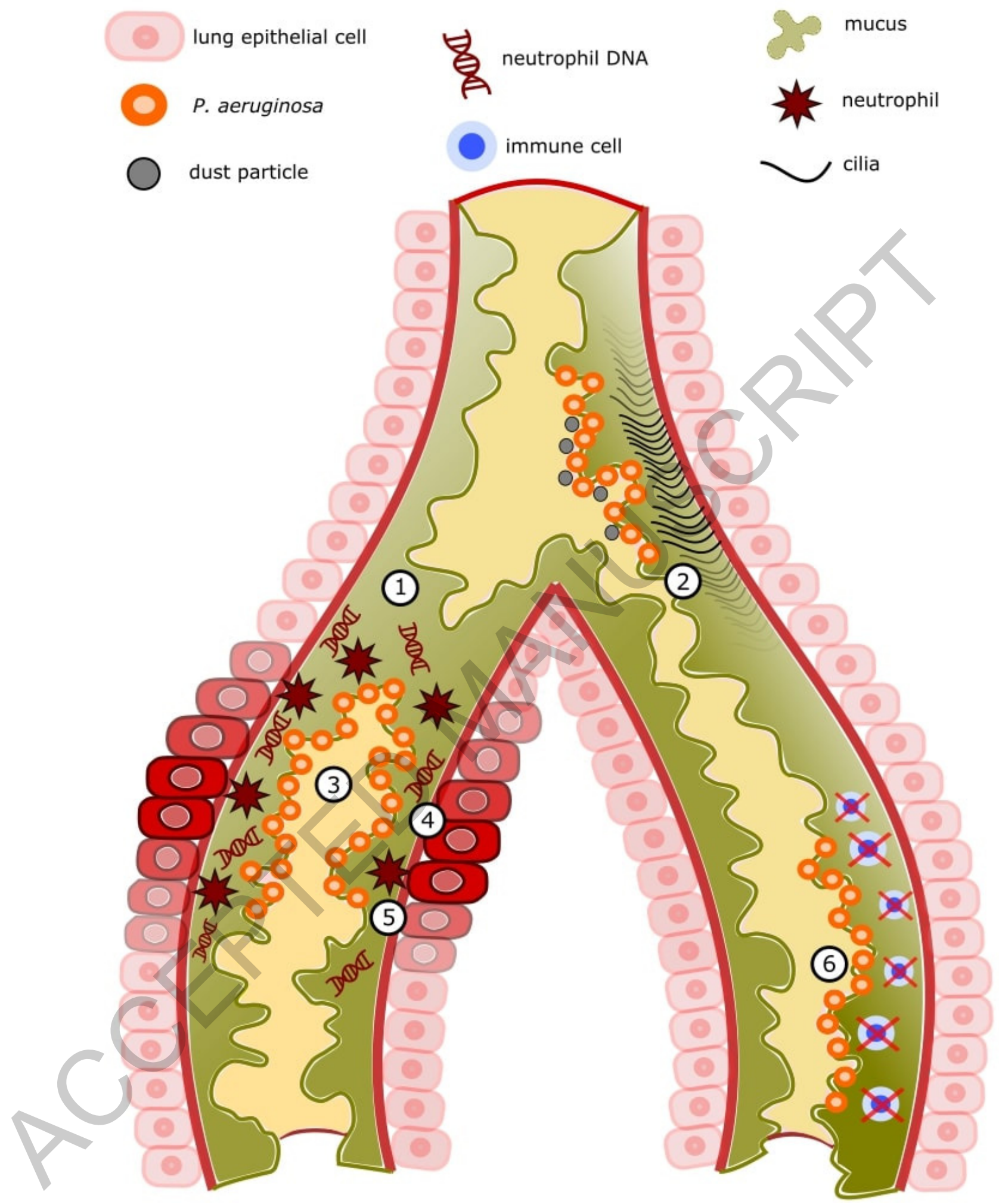

Fig 1 